\title{
Role of Positivity Constraints in Determining Polarized Parton Densities
}

\author{
Elliot Leader \\ Imperial College London \\ Prince Consort Road, London SW' 2BW, England \\ Aleksander V. Sidorov \\ Bogoliubov Theoretical Laboratory \\ Joint Institute for Nuclear Research, 141980 Dubna, Russia \\ Dimiter B. Stamenov \\ Institute for Nuclear Research and Nuclear Energy \\ Bulgarian Academy of Sciences \\ Blvd. Tsarigradsko Chaussee 72, Sofia 1784, Bulgaria
}

\begin{abstract}
We have re-analyzed the world data on inclusive polarized DIS, in both NLO and LO QCD, including the very precise JLab Hall A neutron data, and have studied the role of positivity constraints, and demonstrated their importance, in determining the strange and gluon densities. We have shown that higher twist corrections are essential in the analysis of the present data on the structure function $g_{1}$. A consistent QCD analysis is achieved, and results for the polarized parton densities are given in both the $\overline{\mathrm{MS}}$ and JET schemes.
\end{abstract}




\section{Introduction}

Spurred on by the famous European Muon Collaboration (EMC) experiment [1] at CERN in 1987, there has been a huge growth of interest in the partonic spin structure of the nucleon, i.e., how the nucleon spin is built up out from the intrinsic spin and orbital angular momentum of its constituents, quarks and gluons. Our present knowledge about the spin structure of the nucleon comes from polarized inclusive and semiinclusive DIS experiments at SLAC, CERN, DESY and JLab, polarized proton-proton collisions at RHIC and polarized photoproduction experiments. The determination of the longitudinal polarized parton densities in QCD is one of the important aspects of this knowledge.

In this paper we present an updated version of our NLO QCD polarized parton densities in both the $\overline{\mathrm{MS}}$ and the JET (or so-called chirally invariant) [2] factorization schemes, as well as the LO ones, determined from the world data $[1,3,4]$ on inclusive polarized DIS. Comparing to our previous analyses $[5,6]$ : i) The recent very precise JLab/ Hall A data [4] on $g_{1}^{n} / F_{1}^{n}$ are incorporated into the analysis and ii) New positivity constraints are imposed and their role in the determination of the polarized parton densities is discussed. The updated polarized parton densities (PPD) are compared to those obtained by the other groups and the effect of different positivity constraints that have been imposed is demonstrated.

\section{QCD fits to the data}

In QCD the spin structure function $g_{1}$ can be written in the following form $\left(Q^{2}>>\Lambda^{2}\right)$ :

$$
g_{1}\left(x, Q^{2}\right)=g_{1}\left(x, Q^{2}\right)_{\mathrm{LT}}+g_{1}\left(x, Q^{2}\right)_{\mathrm{HT}},
$$

where "LT" denotes the leading twist $(\tau=2)$ contribution to $g_{1}$, while "HT" denotes the contribution to $g_{1}$ arising from QCD operators of higher twist, namely $\tau \geq 3$. In (1) we have dropped the nucleon target label N. The HT power corrections (up to $\mathcal{O}\left(1 / Q^{2}\right)$ terms $)$ can be divided into two parts:

$$
g_{1}\left(x, Q^{2}\right)_{\mathrm{HT}}=h^{\mathrm{TMC}}\left(x, Q^{2}\right) / Q^{2}+h\left(x, Q^{2}\right) / Q^{2}
$$

where $h^{\mathrm{TMC}}\left(x, Q^{2}\right)$ are the calculable [7] kinematic target mass corrections and effectively belong to the LT term. $h\left(x, Q^{2}\right)$ are the dynamical higher twist $(\tau=3$ and $\tau=4$ ) corrections to $g_{1}$, which are related to multi-parton correlations in the nucleon. The latter are non-perturbative effects and cannot be calculated without using models. 
(Note that the twist-3 contribution to $g_{1}$ is equal to zero if the Wandzura-Wilczek approximation [8] for the spin structure function $g_{2}$ is used.) $g_{1}\left(x, Q^{2}\right)_{\mathrm{LT}}$ in (1) is the well known pQCD expression and in NLO has the form

$$
g_{1}\left(x, Q^{2}\right)_{\mathrm{pQCD}}=\frac{1}{2} \sum_{q}^{N_{f}} e_{q}^{2}\left[(\Delta q+\Delta \bar{q}) \otimes\left(1+\frac{\alpha_{s}\left(Q^{2}\right)}{2 \pi} \delta C_{q}\right)+\frac{\alpha_{s}\left(Q^{2}\right)}{2 \pi} \Delta G \otimes \frac{\delta C_{G}}{N_{f}}\right],
$$

where $\Delta q\left(x, Q^{2}\right), \Delta \bar{q}\left(x, Q^{2}\right)$ and $\Delta G\left(x, Q^{2}\right)$ are quark, anti-quark and gluon polarized densities in the proton, which evolve in $Q^{2}$ according to the spin-dependent NLO DGLAP equations. $\delta C(x)_{q, G}$ are the NLO spin-dependent Wilson coefficient functions and the symbol $\otimes$ denotes the usual convolution in Bjorken $x$ space. $\mathrm{N}_{\mathrm{f}}$ is the number of active flavors. Note that in the LO QCD approximation the coefficients $\delta C(x)_{q, G}$ in (3) vanish and the polarized parton densities evolve according the LO DGLAP equations.

One of the features of polarized DIS is that a lot of the present data are in the preasymptotic region $\left(Q^{2} \sim 1-5 \mathrm{GeV}^{2}, 4 \mathrm{GeV}^{2}<W^{2}<10 \mathrm{GeV}^{2}\right)$. While in the unpolarized case we can cut the low $Q^{2}$ and $W^{2}$ data in order to minimize the less well known higher twist effects, it is impossible to perform such a procedure for the present data on the spin-dependent structure functions without losing too much information. This is especially the case for the HERMES, SLAC and Jefferson Lab experiments. So, to confront correctly the QCD predictions with the experimental data and to determine the polarized parton densities special attention must be paid to the non-perturbative higher twist (powers in $1 / Q^{2}$ ) corrections to the nucleon structure functions.

We have used two approaches to extract the polarized parton densities from the world polarized DIS data. According to the first [5] the leading twist LO/NLO QCD expressions for the structure functions $g_{1}$ and $F_{1}$ have been used in order to confront the data on spin asymmetry $A_{1}\left(\approx g_{1} / F_{1}\right)$ and $g_{1} / F_{1}$. We will refer to these as ' $g_{1} / F_{1}$ ' fits. We have shown $[9,10]$ that in this case the extracted from the world data 'effective' HT corrections $h^{g_{1} / F_{1}}(x)$ to the ratio $g_{1} / F_{1}$

$$
\left[\frac{g_{1}\left(x, Q^{2}\right)}{F_{1}\left(x, Q^{2}\right)}\right]_{e x p} \Leftrightarrow \frac{g_{1}\left(x, Q^{2}\right)_{\mathrm{LT}}}{F_{1}\left(x, Q^{2}\right)_{\mathrm{LT}}}+\frac{h^{g_{1} / F_{1}}(x)}{Q^{2}}
$$

are negligible and consistent with zero within the errors, i.e. $h^{g_{1} / F_{1}}(x) \approx 0$, when for $\left(g_{1}\right)_{L T}$ and $\left(F_{1}\right)_{L T}$ their NLO QCD approximations are used. (Note that in QCD the unpolarized structure function $F_{1}$ takes the same form as $g_{1}$ in $(1)$, namely $F_{1}=$ $\left(F_{1}\right)_{L T}+\left(F_{1}\right)_{H T}$. $)$ What follows from this result is that the higher twist corrections to $g_{1}$ and $F_{1}$ approximately compensate each other in the ratio $g_{1} / F_{1}$ and the NLO PPDs extracted this way are less sensitive to higher twist effects. This is not true in the LO 
case (see our discussion in Ref. [6]). The set of polarized parton densities extracted this way is referred to as $\mathrm{PD}\left(g_{1}^{\mathrm{NLO}} / F_{1}^{\mathrm{NLO}}\right)$ or $\mathrm{PD}($ Set 1$)$.

According to the second approach [6], the $g_{1} / F_{1}$ and $A_{1}$ data have been fitted using phenomenological parametrizations of the experimental data for the unpolarized structure function $F_{2}\left(x, Q^{2}\right)$ and the ratio $R\left(x, Q^{2}\right)$ of the longitudinal to transverse $\gamma N$ cross-sections (i.e. $F_{1}$ is replaced by its expression in terms of usually extracted from unpolarized DIS experiments $F_{2}$ and $R$ ). Note that such a procedure is equivalent to a fit to $\left(g_{1}\right)_{\text {exp }}$, but it is more consistent than the fit to the $g_{1}$ data themselves actually presented by the experimental groups because here the $g_{1}$ data are extracted in the same way for all of the data sets. In this case the HT corrections to $g_{1}$ cannot be compensated because the HT corrections to $F_{1}\left(F_{2}\right.$ and $\left.R\right)$ are absorbed in the phenomenological parametrizations of the data on $F_{2}$ and $R$. Therefore, to extract correctly the polarized parton densities from the $g_{1}$ data, the HT corrections (2) to $g_{1}$ have to be taken into account. In our fit to the data we have used the following expressions for $g_{1} / F_{1}$ and $A_{1}$ :

$$
\begin{aligned}
{\left[\frac{g_{1}^{N}\left(x, Q^{2}\right)}{F_{1}^{N}\left(x, Q^{2}\right)}\right]_{\text {exp }} } & \Leftrightarrow \frac{g_{1}^{N}\left(x, Q^{2}\right)_{\mathrm{LT}}+h^{N}(x) / Q^{2}}{F_{2}^{N}\left(x, Q^{2}\right)_{\exp }} 2 x \frac{\left[1+R\left(x, Q^{2}\right)_{\exp }\right]}{\left(1+\gamma^{2}\right)} \\
A_{1}^{N}\left(x, Q^{2}\right)_{\exp } & \Leftrightarrow \frac{g_{1}^{N}\left(x, Q^{2}\right)_{\mathrm{LT}}+h^{N}(x) / Q^{2}}{F_{2}^{N}\left(x, Q^{2}\right)_{\exp }} 2 x\left[1+R\left(x, Q^{2}\right)_{\exp }\right]
\end{aligned}
$$

where $g_{1}^{N}\left(x, Q^{2}\right)_{\mathrm{LT}}(\mathrm{N}=\mathrm{p}, \mathrm{n}, \mathrm{d})$ is given by the leading twist expression (3) in LO/NLO approximation including the target mass corrections through the Nachtmann variable $[11,12]$. In $(5) h^{N}(x)$ are the dynamical $\tau=3$ and $\tau=4$ HT corrections which are extracted in a model independent way. In our analysis their $Q^{2}$ dependence is neglected. It is small and the accuracy of the present data does not allow to determine it. For the unpolarized structure functions $F_{2}^{N}\left(x, Q^{2}\right)_{\exp }$ and $R\left(x, Q^{2}\right)_{\exp }$ we have used the NMC parametrization [13] and the SLAC parametrization $R_{1998}$ [14], respectively. We will refer to these as ' $\left(g_{1}+\mathrm{HT}\right)$ ' fits, and the set of polarized parton densities extracted according to this approach - $\mathrm{PD}\left(g_{1}^{\mathrm{LT}}+\mathrm{HT}\right)$ or $\mathrm{PD}($ Set 2$)$.

As in our previous analyses [5, 6], for the input LO and NLO polarized parton densities at $Q_{0}^{2}=1 \mathrm{GeV}^{2}$ we have adopted a simple parametrization

$$
\begin{aligned}
x \Delta u_{v}\left(x, Q_{0}^{2}\right) & =\eta_{u} A_{u} x^{a_{u}} x u_{v}\left(x, Q_{0}^{2}\right), \\
x \Delta d_{v}\left(x, Q_{0}^{2}\right) & =\eta_{d} A_{d} x^{a_{d}} x d_{v}\left(x, Q_{0}^{2}\right), \\
x \Delta s\left(x, Q_{0}^{2}\right) & =\eta_{s} A_{s} x^{a_{s}} x s\left(x, Q_{0}^{2}\right), \\
x \Delta G\left(x, Q_{0}^{2}\right) & =\eta_{g} A_{g} x^{a_{g}} x G\left(x, Q_{0}^{2}\right),
\end{aligned}
$$


where on RHS of (6) we have used the MRST98 (central gluon) [15] and MRST99 (central gluon) [16] parametrizations for the LO and $\mathrm{NLO}(\overline{\mathrm{MS}})$ unpolarized densities, respectively. The normalization factors $A_{i}$ in (6) are fixed such that $\eta_{i}$ are the first moments of the polarized densities. To fit better the data in LO QCD, an additional factor $\left(1+\gamma_{v} x\right)$ on the RHS is used for the valence quarks. Bearing in mind that the light quark sea densities $\Delta \bar{u}$ and $\Delta \bar{d}$ cannot, in principle, be determined from the present inclusive data (in the absence of polararized charged current neutrino experiments) we have adopted the convention of a flavor symmetric sea

$$
\Delta u_{\text {sea }}=\Delta \bar{u}=\Delta d_{\text {sea }}=\Delta \bar{d}=\Delta s=\Delta \bar{s}
$$

The first moments of the valence quark densities $\eta_{u}$ and $\eta_{d}$ are constrained by the sum rules

$$
\begin{gathered}
a_{3}=g_{A}=\mathrm{F}+\mathrm{D}=1.2670 \pm 0.0035[17], \\
a_{8}=3 \mathrm{~F}-\mathrm{D}=0.585 \pm 0.025[18],
\end{gathered}
$$

where $a_{3}$ and $a_{8}$ are non-singlet combinations of the first moments of the polarized parton densities corresponding to $3^{\text {rd }}$ and $8^{\text {th }}$ components of the axial vector Cabibbo current

$$
\begin{gathered}
a_{3}=(\Delta u+\Delta \bar{u})\left(Q^{2}\right)-(\Delta d+\Delta \bar{d})\left(Q^{2}\right) \\
a_{8}=(\Delta u+\Delta \bar{u})\left(Q^{2}\right)+(\Delta d+\Delta \bar{d})\left(Q^{2}\right)-2(\Delta s+\Delta \bar{s})\left(Q^{2}\right) .
\end{gathered}
$$

The polarized parton densities (6) and (7) have to satisfy the positivity condition, which in LO QCD implies:

$$
\left|\Delta f_{i}\left(x, Q_{0}^{2}\right)\right| \leq f_{i}\left(x, Q_{0}^{2}\right), \quad\left|\Delta \bar{f}_{i}\left(x, Q_{0}^{2}\right)\right| \leq \bar{f}_{i}\left(x, Q_{0}^{2}\right)
$$

The constraints (12) are the consequence of a probabilistic interpretation of the parton densities in the naive parton model, which is still valid in LO QCD. Beyond LO the parton densities are not physical quantities and the positivity constraints on the polarized parton densities are more complicated. They follow from the positivity condition for the polarized lepton-hadron cross-sections $\Delta \sigma_{i}$ in terms of the unpolarized ones $\left(\left|\Delta \sigma_{i}\right| \leq \sigma_{i}\right)$ and include also the Wilson coefficient functions. It was shown [19], however, that for all practical purposes it is enough, at the present stage, to consider LO positivity bounds for LO as well as for for NLO parton densities, since NLO corrections are only relevant at the level of accuracy of a few percent.

While in our previous NLO QCD analyses we have mainly used for the unpolarized parton densities on the RHS of (12) the Barone et al. parametrization [20] we are here using the MRST02(NLO) updated unpolarized parton densities [21] in both $\overline{\mathrm{MS}}$ and JET schemes. The only significant change in the MRST02 NLO partons, compared to 
Table 1. The parameters of the Set 1 of NLO input parton polarized densities $\mathrm{PD}\left(g_{1}^{\mathrm{NLO}} / F_{1}^{\mathrm{NLO}}\right)$ at $Q^{2}=1 \mathrm{GeV}^{2}$ as obtained from the fits to the world $[1,3]$ and JLab [4] data in the $\overline{\mathrm{MS}}$ and JET schemes. The errors shown are total (statistical and systematic). The parameters marked by $\left(^{*}\right)$ are fixed by the sum rules (8) and (9).

\begin{tabular}{|c|c|c|}
\hline Fit & $g_{1}^{\mathrm{NLO}} / F_{1}^{\mathrm{NLO}}(\overline{\mathrm{MS}})$ & $g_{1}^{\mathrm{NLO}} / F_{1}^{\mathrm{NLO}}(\mathrm{JET})$ \\
\hline $\mathrm{DF}$ & $188-6$ & $188-6$ \\
$\chi^{2}$ & 159.0 & 158.5 \\
$\chi^{2} / \mathrm{DF}$ & 0.874 & 0.871 \\
\hline$\eta_{u}$ & $0.926^{*}$ & $0.926^{*}$ \\
$a_{u}$ & $0.209 \pm 0.018$ & $0.211 \pm 0.018$ \\
$\eta_{d}$ & $-0.341^{*}$ & $-0.341^{*}$ \\
$a_{d}$ & $0.072 \pm 0.068$ & $0.083 \pm 0.058$ \\
$\eta_{s}$ & $-0.066 \pm 0.009$ & $-0.049 \pm 0.013$ \\
$a_{s}$ & $0.649 \pm 0.117$ & $0.779 \pm 0.214$ \\
$\eta_{g}$ & $0.195 \pm 0.257$ & $0.289 \pm 0.316$ \\
$a_{g}$ & $2.575 \pm 1.729$ & $0.000 \pm 0.686$ \\
\hline
\end{tabular}

those of MRST99 (which we have also used in some of our analyses), is in the gluon, and especially in an increase in the gluon density at high $\mathrm{x}$. In the MRST02 fit to the world data the authors no longer include prompt photon data due to theoretical problems and possible inconsistencies between data sets, and instead allow the high $x$ gluon to be determined by the improved Tevatron jet data [22], which considerably improves the determination of the gluon. The use of new positivity constraints leads to a significant change in the polarized strange quark and gluon densities, the importance of which will be discussed further.

\section{Results}

In this section we present the numerical results of our fits to the world data $[1,3]$ on $g_{1} / F_{1}$ and $A_{1}$ including JLab Hall A neutron data [4]. The data used (188 experimental points) cover the following kinematic region:

$$
0.005 \leq x \leq 0.75, \quad 1<Q^{2} \leq 58 \mathrm{GeV}^{2}
$$


Table 2. The parameters of the Set $\mathbf{2}$ of LO, NLO $(\overline{\mathrm{MS}})$ and $\mathrm{NLO}(\mathrm{JET})$ input parton densities $\mathrm{PD}\left(g_{1}^{\mathrm{LT}}+\mathrm{HT}\right)$ at $Q^{2}=1 G e V^{2}$ as obtained from the best $\left(g_{1}+\mathrm{HT}\right)$ fits to the world $[1,3]$ and JLab [4] data. The errors shown are total (statistical and systematic). The parameters marked by $\left(^{*}\right)$ are fixed. Note that the TMC are included in $\left(g_{1}\right)_{\mathrm{LT}}$.

\begin{tabular}{|c|c|c|c|}
\hline Fit & $\left(g_{1}\right)_{\mathrm{LO}}+h(x) / Q^{2}$ & $\left(g_{1}\right)_{\mathrm{NLO}(\overline{\mathrm{MS}})}+h(x) / Q^{2}$ & $\left(g_{1}\right)_{\mathrm{NLO}(\mathrm{JET})}+h(x) / Q^{2}$ \\
\hline $\mathrm{DF}$ & $188-16$ & $188-16$ & $188-16$ \\
\hline$\chi^{2}$ & 152.7 & 150.0 & 150.8 \\
\hline$\chi^{2} / \mathrm{DF}$ & 0.888 & 0.872 & 0.877 \\
\hline$\eta_{u}$ & $0.926^{*}$ & $0.926^{*}$ & $0.926^{*}$ \\
\hline$a_{u}$ & $0.000 \pm 0.011$ & $0.244 \pm 0.036$ & $0.240 \pm 0.037$ \\
\hline$\gamma_{u}$ & $1.607 \pm 0.292$ & $0^{*}$ & $0^{*}$ \\
\hline$\eta_{d}$ & $-0.341^{*}$ & $-0.341^{*}$ & $-0.341^{*}$ \\
\hline$a_{d}$ & $0.000 \pm 0.067$ & $0.123 \pm 0.134$ & $0.120 \pm 0.148$ \\
\hline$\gamma_{d}$ & $3.184 \pm 1.630$ & $0^{*}$ & $0^{*}$ \\
\hline$\eta_{s}$ & $-0.075 \pm 0.010$ & $-0.078 \pm 0.012$ & $-0.064 \pm 0.017$ \\
\hline$a_{s}$ & $0.514 \pm 0.068$ & $0.629 \pm 0.090$ & $0.612 \pm 0.121$ \\
\hline$\eta_{g}$ & $0.602^{*}$ & $0.348 \pm 0.345$ & $0.268 \pm 0.422$ \\
\hline$a_{g}$ & $0.328^{*}$ & $1.980 \pm 1.359$ & $2.851 \pm 1.444$ \\
\hline$x_{i}$ & \multicolumn{3}{|c|}{$h^{p}\left(x_{i}\right)\left[G e V^{2}\right]$} \\
\hline 0.028 & $-0.003 \pm 0.036$ & $0.010 \pm 0.042$ & $0.024 \pm 0.039$ \\
\hline 0.100 & $-0.097 \pm 0.033$ & $-0.043 \pm 0.034$ & $-0.040 \pm 0.039$ \\
\hline 0.200 & $-0.164 \pm 0.032$ & $-0.106 \pm 0.036$ & $-0.108 \pm 0.038$ \\
\hline 0.350 & $-0.036 \pm 0.036$ & $-0.016 \pm 0.038$ & $-0.015 \pm 0.040$ \\
\hline 0.600 & $0.032 \pm 0.019$ & $0.046 \pm 0.019$ & $0.048 \pm 0.019$ \\
\hline$x_{i}$ & \multicolumn{3}{|c|}{$h^{n}\left(x_{i}\right)\left[G e V^{2}\right]$} \\
\hline 0.028 & $0.204 \pm 0.078$ & $0.145 \pm 0.081$ & $0.161 \pm 0.081$ \\
\hline 0.100 & $0.168 \pm 0.050$ & $0.192 \pm 0.047$ & $0.197 \pm 0.046$ \\
\hline 0.200 & $0.023 \pm 0.058$ & $0.035 \pm 0.067$ & $0.032 \pm 0.070$ \\
\hline 0.325 & $0.031 \pm 0.027$ & $0.019 \pm 0.031$ & $0.018 \pm 0.035$ \\
\hline 0.500 & $0.031 \pm 0.015$ & $0.010 \pm 0.014$ & $0.011 \pm 0.016$ \\
\hline
\end{tabular}


The total (statistical and systematic) errors are taken into account. The systematic errors are added quadratically.

We have determined from the data two sets of polarized parton densities in both the $\overline{\mathrm{MS}}$ and the JET factorization schemes: $\operatorname{PD}\left(g_{1}^{\mathrm{NLO}} / F_{1}^{\mathrm{NLO}}\right)$ (see Table 1$)$ and $\operatorname{PD}\left(g_{1}^{\mathrm{LT}}+\right.$ HT) (see Table 2). For the second set of polarized parton densities a LO version $\mathrm{PD}\left(g_{1}^{\mathrm{LO}}+\mathrm{HT}\right)$ is also presented. The latter have been extracted from the data using for the unpolarized parton densities on the RHS of the positivity bounds (12) the LO MRST'01 ones (the MRST'02 LO set is essentially identical to the MRST'01 one and is not presented by the authors). Note once more that in the LO ' $g_{1} / F_{1}$ ' fit to the data the polarized parton densities $\mathrm{PD}\left(g_{1}^{\mathrm{LO}} / F_{1}^{\mathrm{LO}}\right)$ cannot be correctly determined because the Callan-Gross relation $2 x F_{1}\left(x, Q^{2}\right)_{\mathrm{LO}}=F_{2}\left(x, Q^{2}\right)_{\mathrm{LO}}$ (used in the calculation of $F_{1}$ ) is strongly broken (by up to $30 \%$ ) for $x<0.25$ and small $Q^{2}$. In other words, to extract correctly the LO polarized parton densities from the data, the second method of analysis have to be used, i.e. the high twist corrections to $g_{1}$ have to be taken into account. This observation is especially important for the analysis of the semi-inclusive DIS data, where the LO QCD approximation is mainly used.
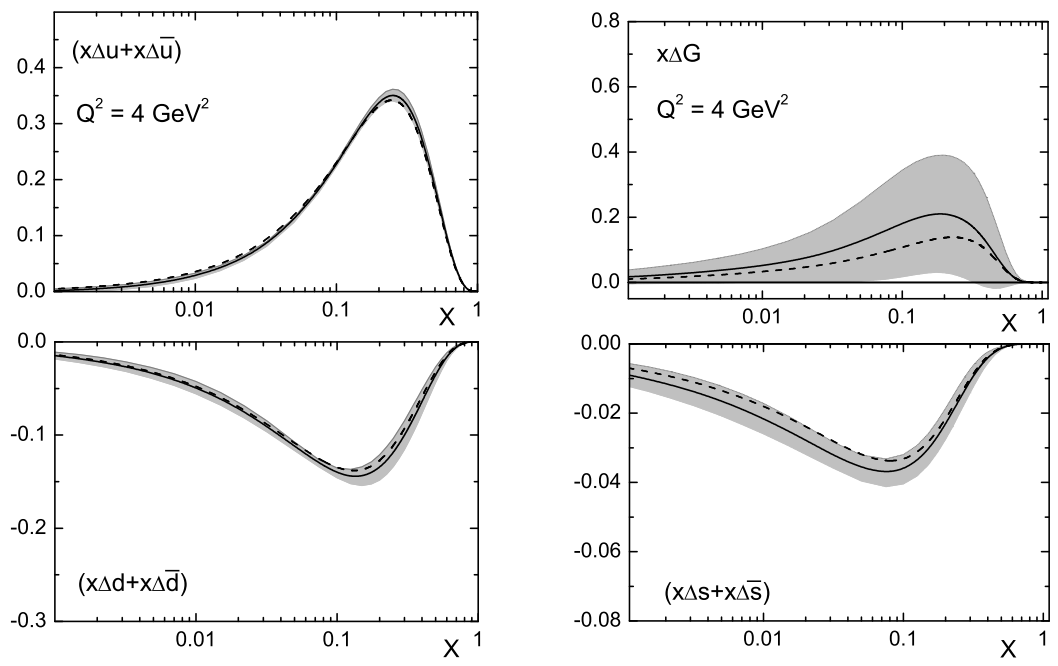

Figure 1: $\operatorname{NLO}(\overline{\mathrm{MS}})$ polarized parton densities $\mathrm{PD}\left(g_{1}^{\mathrm{LT}}+\mathrm{HT}\right)$ (solid curves) together with their error bands compared to $\mathrm{PD}\left(g_{1}^{\mathrm{NLO}} / F_{1}^{\mathrm{NLO}}\right)$ (dashed curves) at $Q^{2}=4 \mathrm{GeV}^{2}$.

In Fig. 1 we compare the $\mathrm{NLO}(\overline{\mathrm{MS}})$ polarized parton densities $\mathrm{PD}\left(g_{1}^{\mathrm{LT}}+\mathrm{HT}\right)$ with $\mathrm{PD}\left(g_{1}^{\mathrm{NLO}} / F_{1}^{\mathrm{NLO}}\right)$. As seen from Fig. 1 the two sets of polarized parton densities are very close to each other, especially for $u$ and $d$ quarks. This is a good illustration of the fact that a fit to the $g_{1}$ data taking into account the higher twist corrections to $g_{1}$ 
$\left(\chi_{\mathrm{DF}, \mathrm{NLO}}^{2}=0.872\right)$ is equivalent to a fit of the data on $A_{1}\left(\sim g_{1} / F_{1}\right)$ and $g_{1} / F_{1}$ using for the $g_{1}$ and $F_{1}$ structure functions their NLO leading twist expressions $\left(\chi_{\mathrm{DF}, \mathrm{NLO}}^{2}=\right.$ 0.874). In other words, this fact confirms once more that the higher twist corrections to $g_{1}$ and $F_{1}$ approximately cancel in the ratio $g_{1} / F_{1}$. Nevertheless, we consider that the Set 2 of the polarized parton densities $\operatorname{PD}\left(g_{1}^{\mathrm{LT}}+\mathrm{HT}\right)$ is preferable because using them and simultaneously extracted higher twist corrections to $g_{1}$, the spin structure function $g_{1}$ can be correctly calculated in the preasymptotic $\left(Q^{2}, W^{2}\right)$ region too.
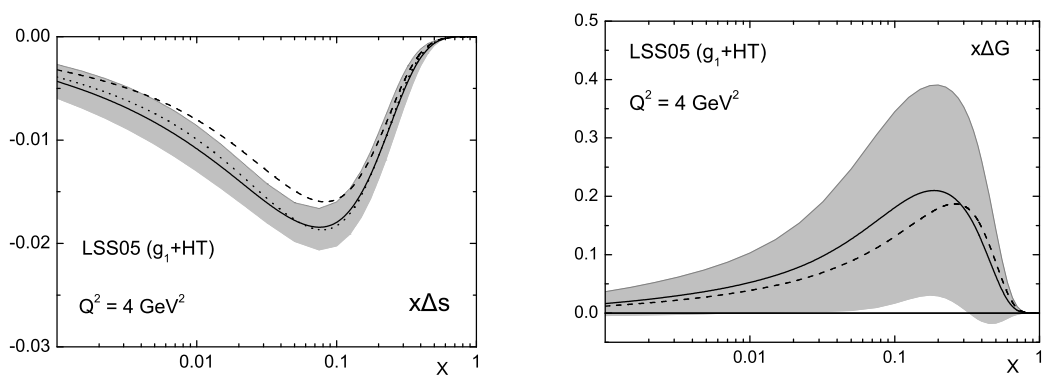

Figure 2: Comparison between NLO Set 2 polarized strange sea and gluon densities at $Q^{2}=4 \mathrm{GeV}^{2}$ in the $\overline{\mathrm{MS}}$ (solid curves) and the JET scheme (dashed curves). Dot curve corresponds to $\Delta s\left(x, Q^{2}\right)_{\mathrm{JET}} \Rightarrow \overline{\mathrm{MS}}$ obtained by the transformation rule (14) (see the text).

Let us briefly discuss the scheme dependence of our results. Recall that according to perturbative QCD the NLO polarized valence quarks and gluons should be the same [up to order of $\mathcal{O}\left(\alpha_{s}^{3}\right)$ ] in both the $\overline{\mathrm{MS}}$ and the JET factorization schemes, while for the moments of the strange sea quarks the following transformation rule is valid:

$$
\Delta s\left(n, Q^{2}\right)_{\overline{\mathrm{MS}}}=\Delta s\left(n, Q^{2}\right)_{\mathrm{JET}}-\frac{\alpha_{s}\left(Q^{2}\right)}{2 \pi n(n+1)} \Delta G\left(n, Q^{2}\right)_{\mathrm{JET}} .
$$

It is seen from the Table 1 and Table 2 that the values of $\chi^{2} / \mathrm{DF}(\overline{\mathrm{MS}})$ and $\chi^{2} / \mathrm{DF}(\mathrm{JET})$ coincide almost exactly for the $g_{1} / F_{1}$ as well as for the $\left(g_{1}^{\mathrm{LT}}+\mathrm{HT}\right)$ fits, which is a good indication of the stability of the analysis regardless of the scheme used. To illustrate the factorization scheme dependence, the extracted polarized $\operatorname{PD}\left(g_{1}^{\mathrm{NLO}}+\right.$ HT) in the schemes under consideration are compared in Fig. 2. Note that the valence densities $\Delta u_{v}$ and $\Delta d_{v}$ in the $\overline{\mathrm{MS}}$ and JET schemes are almost identical (see the values of the parameters for the corresponding input parton densities in Table 2) and in excellent agreement with what follows from QCD. So the corresponding curves are not shown in Fig. 2. The extracted polarized gluons in the two schemes are also 
well consistent within the errors (see Fig. 2). In Fig. 2 we also show the polarized strange sea densities in both schemes, determined directly from the fits and evolution equations, as well as the strange sea $\Delta s\left(x, Q^{2}\right)_{\mathrm{JET}} \Rightarrow \overline{\mathrm{MS}}$ obtained by the transformation rule (14). It is seen that: i) at large $x \Delta s\left(x, Q^{2}\right)_{\overline{\mathrm{MS}}}$ is very close to $\Delta s\left(x, Q^{2}\right)_{\mathrm{JET}}$ which is consistent with (14), since large $n$ in the Mellin space corresponds to large Bjorken $x$, and ii) $\Delta s\left(x, Q^{2}\right)_{\mathrm{JET} \Rightarrow \overline{\mathrm{MS}}}$ coincides very well with $\Delta s\left(x, Q^{2}\right) \overline{\mathrm{MS}}$. We have discussed here in detail the results on the set $\operatorname{PD}\left(g_{1}^{\mathrm{NLO}}+\mathrm{HT}\right)$. We have found the same conclusion for the other set of polarized parton densities $\operatorname{PD}\left(g_{1}^{\mathrm{NLO}} / F_{1}^{\mathrm{NLO}}\right)$. In conclusion, we have found that the obtained numerical results are in a good agreement with the perturbative QCD predictions.

Compared to our previous results $[5,6]$ we now obtain smaller values for the gluon polarization (the first moment of $\Delta G\left(x, Q^{2}\right)$ ), which leads to a smaller difference between the values of the strange quark polarization (the first moment of $\Delta s\left(x, Q^{2}\right)$ ) determined in the $\overline{\mathrm{MS}}$ and the JET schemes, respectively. To illustrate this tendency we present in Table 3 the corresponding values of the first moments, $\Delta s, \Delta G$ and $\Delta \Sigma$, for the LSS'01 and LSS'05(Set 1) sets of polarized parton densities. Note that in the JET scheme the singlet polarization $\Delta \Sigma\left(Q^{2}\right)$ is a $Q^{2}$ independent quantity. Then, in this scheme it is meaningful to directly interpret $\Delta \Sigma$ as the contribution of the quark spins to the nucleon spin and to compare its value obtained in the DIS region with the predictions of the different (constituent, chiral, etc.) quark models at low $Q^{2}\left(Q^{2} \sim \Lambda^{2}\right)$. Our new value of $\Delta \Sigma_{\mathrm{JET}}=0.29 \pm 0.08$ is smaller then the old one and farther from the value 0.6 of $\Delta \Sigma$ at low $Q^{2}$ region predicted in relativistic constituent quark models [23]. Note, however, that if nonperturbative vacuum spin effects are taken into account $[24,25]$, the value of $\Delta \Sigma$ at low $Q^{2}$ is expected to be smaller than 0.6. Further theoretical and experimental investigation in both the large and very low $Q^{2}$ regions, is needed to answer more precisely the important question as to the fraction of the nucleon spin carried by its quarks.

Table 3. First moments (polarizations) of LSS'01 and LSS'05(Set 1) polarized parton densities at $Q^{2}=1 \mathrm{GeV}^{2}$.

\begin{tabular}{|c|c|c|c|c|}
\hline Fit & $\Delta s\left(Q^{2}\right)_{\overline{\mathrm{MS}}}$ & $\Delta s\left(Q^{2}\right)_{\mathrm{JET}}$ & $\Delta G\left(Q^{2}\right)_{\mathrm{JET}}$ & $\Delta \Sigma_{\text {JET }}$ \\
\hline LSS'01 & $-0.065 \pm 0.016$ & $-0.035 \pm 0.010$ & $0.68 \pm 0.32$ & $0.37 \pm 0.07$ \\
\hline LSS'05/Set 1 & $-0.066 \pm 0.009$ & $-0.049 \pm 0.013$ & $0.29 \pm 0.32$ & $0.29 \pm 0.08$ \\
\hline
\end{tabular}


The extracted higher twist corrections to the proton and neutron spin structure functions, $h^{p}(x)$ and $h^{n}(x)$, are shown in Fig. $3 .^{\dagger}$ As seen from Fig. 3 the size of the
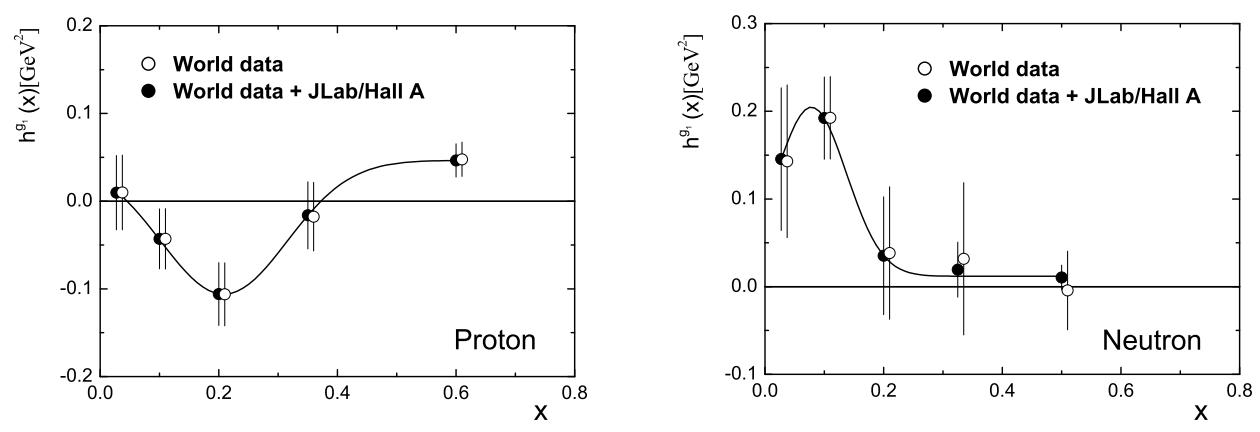

Figure 3: Higher twist corrections to the proton and neutron $g_{1}$ structure functions extracted from the data on $g_{1}$ in $\mathrm{NLO}(\overline{\mathrm{MS}}) \mathrm{QCD}$ approximation for $g_{1}\left(x, Q^{2}\right)_{\mathrm{LT}}$. The parametrization (15) of the higher twist values is also shown.

HT corrections is not negligible and their shape depends on the target. In Fig. 3 our previous results on the higher twist corrections to $g_{1}$ (before the JLab Hall A data were available) are also presented. As seen from Fig. 3, thanks to the very precise JLab Hall A data at large $x$ the higher twist corrections to the neutron spin structure function are now much better determined in this region. In Fig. 3 our parametrizations of the values of higher twists for the proton and neutron targets

$$
\begin{aligned}
& h^{p}(x)=0.0465-\frac{0.1913}{\sqrt{\pi / 2}} \exp \left[-2((x-0.2087) / 0.2122)^{2}\right] \\
& h^{n}(x)=0.0119+\frac{0.2420}{\sqrt{\pi / 2}} \exp \left[-2((x-0.0783) / 0.1186)^{2}\right]
\end{aligned}
$$

are also shown. These should be helpful in a calculation of the nucleon structure function $g_{1}$ for any $x$ and moderate $Q^{2}$ in the experimental region, where the higher twist corrections are not negligible. The values of the higher twist corrections to the proton and neutron $g_{1}$ structure functions extracted in a model independent way from polarized DIS data are in agreement with the QCD sum rule estimates [27] as well as with the instanton model predictions [28] but disagree with the renormalon calculations [29].

\footnotetext{
${ }^{\dagger}$ The moments of higher twist contribution to the proton structure function $g_{1}$, including also in the analysis the data on the resonance region, have been determined in the very recent paper [26].
} 

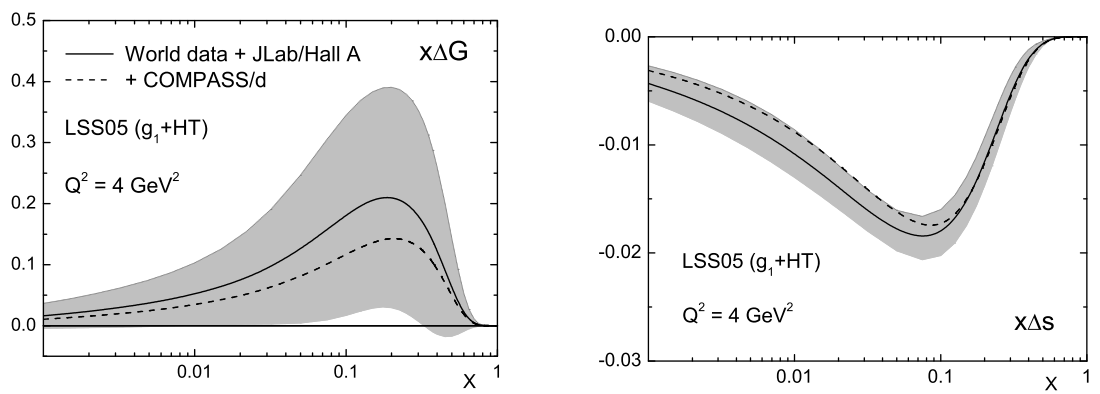

Figure 4: Effect of the COMPASS data on the Set $2 \mathrm{NLO}(\overline{\mathrm{MS}})$ polarized parton densities $\mathrm{PD}\left(g_{1}^{\mathrm{NLO}}+\mathrm{HT}\right)$.

When this analysis was finished, the COMPASS Collaboration at CERN reported new data on the longitudinal asymmetry $A_{1}^{d}$ [30]. Their results improve considerably the statistical accuracy on the small $x$ region $0.004<x<0.03$. We have carried out a preliminary study (' $g_{1}+$ HT' fit in the $\overline{\mathrm{MS}}$ scheme) to see whether the COMPASS data has any significant effect on the result of our analysis. The effect of the new data

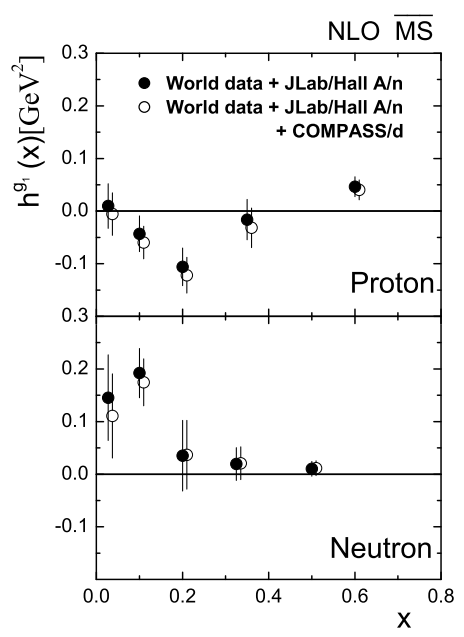

Figure 5: Effect of the COMPASS data on the higher twist values.

on the polarized parton densities $\mathrm{PD}\left(g_{1}^{\mathrm{NLO}}+\mathrm{HT}\right)$ and the higher twist corrections is illustrated in Fig. 4 and Fig. 5. While the valence quarks densities $\Delta u_{v}$ and $\Delta d_{v}$ do not change in the experimental region (for that reason they are not shown in Fig. 4), the magnitudes of both the polarized gluon and strange quark sea densities decrease, but the corresponding curves lie within the error bands (see Fig. 4). The impact of the new data on the values of higher twist corrections is negligible (see Fig. 5). The new 
values are in good agreement with the old ones although there is a tendency for the central values for the proton target to be slightly lower than the old ones. The central values of the HT correction for the neutron at small $x$ are also slightly lower than the old ones.

\section{Impact of positivity constraints on polarized PD}

Let us consider now how the use of different positivity constraints influences the results on the polarized parton densities. In Fig. 6 we compare our new Set 1 of NLO( $\overline{\mathrm{MS}})$ polarized parton densities $\mathrm{PD}\left(g_{1}^{\mathrm{NLO}} / F_{1}^{\mathrm{NLO}}\right)$ with LSS'2001 parton densities [5] presented on the HEPDATA web site. Both sets are determined from the data by the same method but using different positivity constraints. (Note that the inclusion of the JLab data do not influence the results on the polarized parton densities if the same positivity constraints are used.) While the new polarized $\mathrm{PD}\left(g_{1}^{\mathrm{NLO}} / F_{1}^{\mathrm{NLO}}\right)$ are compatible with the positivity bounds (12) imposed by the MRST'02 unpolarized parton densities [21], those of the LSS'2001 set are limited by the Barone et al. unpolarized parton densities [20]. As seen from Fig. 6 the valence quark densities $\Delta u_{v}$ and $\Delta d_{v}$ of the two sets are close to each other, while the polarized strange sea quark and gluon densities are significantly different. This comparison is a good illustration of the fact that the present inclusive polarized DIS data allow a much better determination of the valence quark
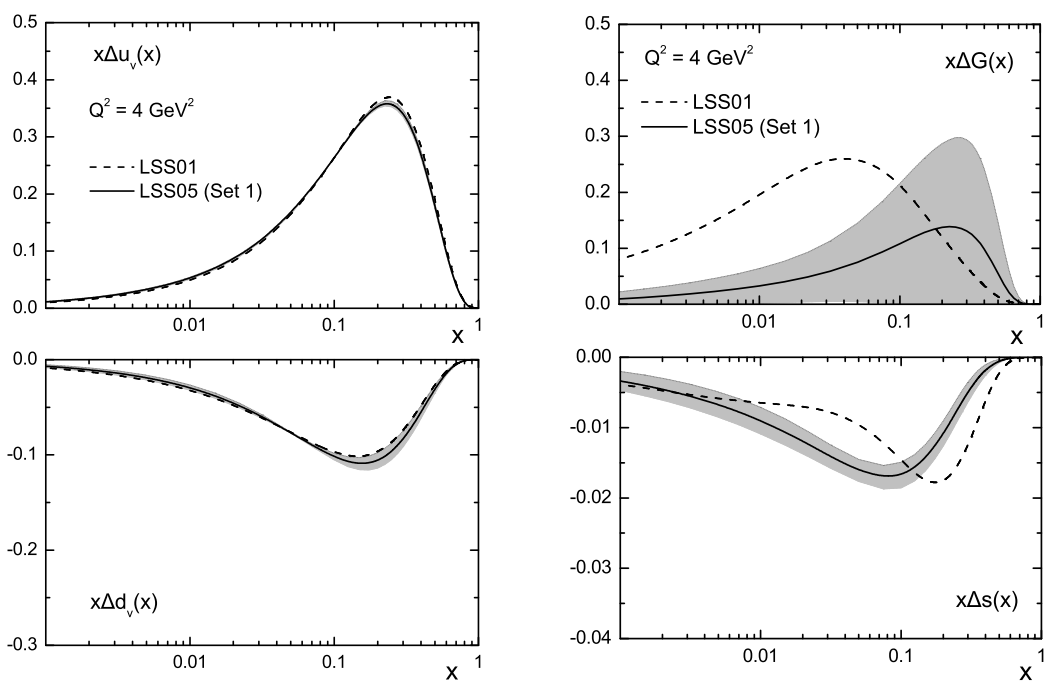

Figure 6: Comparison between our two sets of $\mathrm{NLO}(\overline{\mathrm{MS}})$ polarized parton densities, LSS'01 and LSS'05(Set 1), at $Q^{2}=4 \mathrm{GeV}^{2}$. 

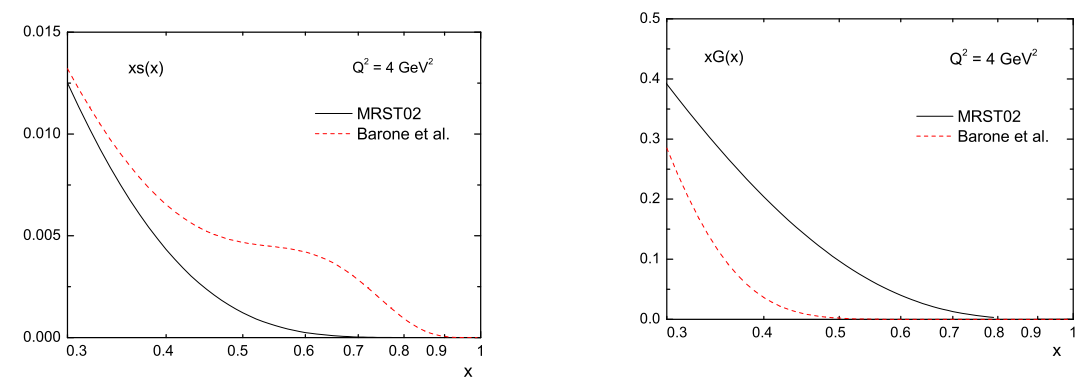

Figure 7: Comparison between the $\operatorname{NLO}(\overline{\mathrm{MS}})$ unpolarized strange quark sea and gluon densities determined by MRST'02 [21] and Barone at al. [20].

densities (if SU(3) symmetry of the flavour decomposition of the sea is assumed) than the polarized strange quarks $\Delta s\left(x, Q^{2}\right)$ and the polarized gluons $\Delta G\left(x, Q^{2}\right)$. This is especially true for the high $x$ region, where the values of $\Delta s\left(x, Q^{2}\right)$ and $\Delta G\left(x, Q^{2}\right)$ are very small and the precision of the data is not enough to extract them correctly. That is why different unpolarized sea quark and gluon densities (see Fig. 7) used on the RHS of the positivity constraints (12) are important and crucial in determining $\Delta s\left(x, Q^{2}\right)$
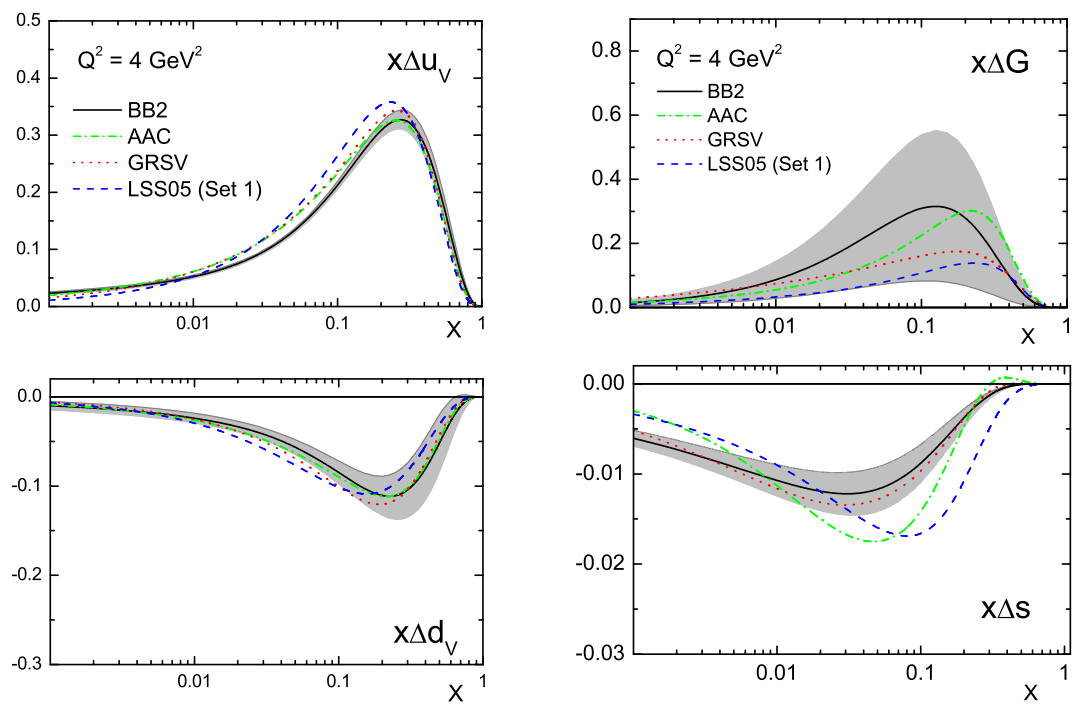

Figure 8: Comparison between our $\operatorname{NLO}(\overline{\mathrm{MS}})$ polarized parton densities (Set 1) at $Q^{2}=4 \mathrm{GeV}^{2}$ with those obtained by GRSV ('standard scenario') [31], BB (ISET=4 or BB2) [32] and AAC (AAC03) [33].

and $\Delta G\left(x, Q^{2}\right)$ in this region. The more restrictive $s\left(x, Q^{2}\right)_{\mathrm{MRST}^{\prime} 02}$ at high $x$ leads to a smaller value of $\left|\Delta s\left(x, Q^{2}\right)\right|_{\text {LSS }^{\prime} 5}$ in this region, while the smaller $G\left(x, Q^{2}\right)$ Bar.et.al pro- 
vides a stronger constraint on $\Delta G\left(x, Q^{2}\right)_{\text {LSS }^{\prime} 1}$ (see Fig. 6). To illustrate this fact once more, we compare our new polarized parton densities $\operatorname{PD}\left(g_{1}^{\mathrm{NLO}} / F_{1}^{\mathrm{NLO}}\right)$ at $Q^{2}=4 G e V^{2}$ with those obtained by GRSV [31], Blumlein, Bottcher [32] and the Asymmetry Analy-
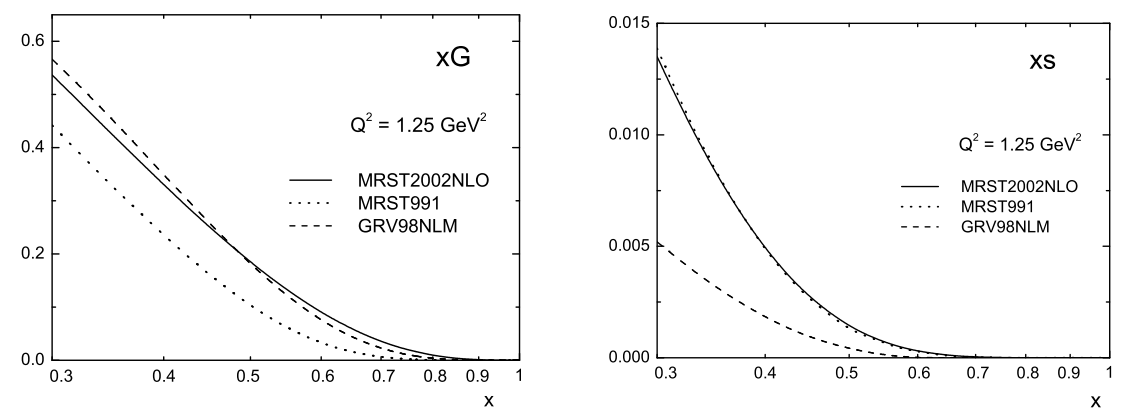

Figure 9: Comparison between the $\operatorname{NLO}(\overline{\mathrm{MS}})$ unpolarized strange quark sea and gluon densities determined by MRST [16, 21] and GRV [34].

sis Collaboration (AAC) [33] using almost the same set of data (see Fig. 8). Note that all these groups have used the GRV unpolarized parton densities [34] for constraining their polarized parton densities at large $x$. As seen from Fig. 9, in this $x$ region the unpolarized GRV and MRST'02 gluons are practically the same, while the magnitude of the unpolarized GRV strange sea quarks is much smaller than that of MRST'02. Therefore, the GRV unpolarized strange sea quarks provide a stronger constraint on the polarized ones. The impact on the determination of the polarized strange sea density is demonstrated in Fig. 10. (The GRV and MRST'02 unpolarized strange sea densities are also shown.) As a result the magnitude of our polarized strange sea density $x\left|\Delta s\left(x, Q^{2}\right)\right|$ is larger in the region $x>0.1$ than those obtained by the other groups. Note also that the magnitude of $x \Delta s$ obtained by the GRSV and BB is smaller than that determined by AAC. We consider the GRSV result to be a consequence of the fact that in their analysis, the GRV positivity constraint is imposed at lower value of $Q^{2}$ : $Q^{2}=\mu_{N L O}^{2}=0.4 \mathrm{GeV}^{2}$, while AAC has used the same requirement at $Q^{2}=1 \mathrm{GeV}^{2}$. Finally, the different positivity conditions on $\Delta s$ influence also the determination of the polarized gluon density for larger $Q^{2}$ because the evolution in $Q^{2}$ mixes the polarized sea quarks and gluons. To end this section we would like to emphasize that for the adequate determination of polarized strange quarks and gluons at large $x$, the role of the corresponding unpolarized densities is very important. That is why the latter have to be determined with good accuracy at large $x$ in the preasymptotic $\left(Q^{2}, W^{2}\right)$ region too. Usually the sets of unpolarized parton densities, presented in the literature, are extracted from the data on DIS using cuts in $Q^{2}$ and $W^{2}$ chosen in order to minimize 
the higher twist effects. In order to use the densities for constraining the polarized parton densities they have to be continued to the preasymptotic $\left(Q^{2}, W^{2}\right)$ region. It is not obvious that the continued unpolarized parton densities would coincide well with those obtained from the data in the region $\left(Q^{2}>1 \mathrm{GeV}^{2}, W^{2}>4 \mathrm{GeV}^{2}\right)$ in the presence of the $\mathrm{HT}$ corrections to unpolarized structure functions $F_{1}$ and $F_{2}$. So, a QCD analysis of the unpolarized world data including the preasymptotic $\left(Q^{2}, W^{2}\right)$ region and taking into account $\mathrm{HT}$ corrections is needed in order to extract correctly the unpolarized parton densities in the preasymptotic region. Our arguments for the

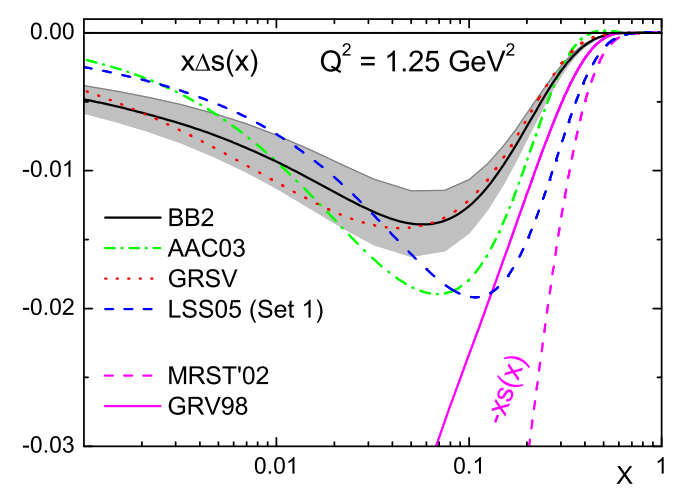

Figure 10: Comparison between our $\operatorname{NLO}(\overline{\mathrm{MS}})$ polarized strange sea quark density (Set 1) at $Q^{2}=1.25 \mathrm{GeV}^{2}$ with those obtained by GRSV ('standard scenario') [31], BB $(\mathrm{ISET}=4$ or BB2) [32] and AAC (AAC03) [33]. The unpolarized MRST02 and GRV98 strange sea quark densities are also shown.

need for a precise determination of the unpolarized densities of strange quarks and gluons in both the asymptotic and preasymptotic regions in $Q^{2}$ and $W^{2}$, coming from spin physics, could be considered as additional to those discussed in the recent paper $[35]$.

\section{Conclusions}

i) We have re-analyzed the world data on inclusive polarized deep inelastic leptonnucleon scattering in leading and next-to-leading order of QCD, adding to the old set of data the very precise JLab Hall A neutron data. Compared to our previous analyses new positivity constraints on the polarized parton densities have been used. The latter reflect mainly the better determination of unpolarized gluon density, especially at high $x$. Two new sets of NLO polarized parton densities in the JET and $\overline{\mathrm{MS}}$ factorization 
schemes as well as LO polarized patron densities have been extracted from the data using different methods of analysis. The NLO polarized parton densities determined in the two schemes are in a good agreement with the pQCD predictions.

ii) The impact of positivity constraints on the polarized parton densities has been studied. Special attention has been paid to the role of positivity constraints in determining the polarized strange quark and gluon densities, which are not well determined from the present inclusive DIS data. For that reason the effect of the positivity conditions used to constrain them is very important. On other hand, the different sets of unpolarized parton densities needed to impose the positivity bounds (12) are usually determined in the DIS region and their continuation to the preasymptotic region via the evolution DGLAP equations sometimes leads to very different behaviour of the unpolarized parton densities belonging to the different sets. In particular, it is demonstrated that the use of MRST'02 and GRV98 NLO( $\overline{\mathrm{MS}})$ unpolarized strange quark densities in the RHS of the positivity condition (12) leads to a significant difference of the extracted polarized strange sea density, especially for $x>0.05$. So, a more precise determination of the unpolarized parton densities in the preasymptotic region is very important for a better determination of the polarized ones, especially of the polarized strange quark sea and gluon densities, which are weakly constrained by the present experimental data.

iii) It was demonstrated that in the fit to the $g_{1}$ data the higher twist corrections to $g_{1}$ are important and have to be taken into account. It was also shown that the values of higher twist corrections to the neutron spin structure function at high $x$ are determined much more precisely when the JLab Hall A data are used in the analysis.

$i v)$ Finally, the use of the very recent COMPASS data on inclusive asymmetry

$A_{1}^{d}$ has little or no effect on the polarized valence densities, but the magnitudes of the strange quark and gluon densities decrease.

\section{Acknowledgments}

This research was supported by the JINR-Bulgaria Collaborative Grant, by the RFBR (No 05-01-00992, 03-02-16816) and by the Bulgarian National Science Foundation under Contract Ph-1010. 


\section{Appendix}

For practical purposes we present here explicitly our Set 1 and Set 2 of polarized parton densities at $Q^{2}=1 \mathrm{GeV}^{2}$. The polarized valence quark densities correspond to $\mathrm{SU}(3)$ flavour symmetric sea.

$$
\begin{aligned}
\operatorname{LSS}^{\prime} 05(\text { Set 1) } & \operatorname{NLO}(\overline{\mathrm{MS}}) \operatorname{PD}\left(g_{1} / F_{1}\right): \\
x \Delta u_{v}(x) & =0.4621 x^{0.6258}(1-x)^{3.428}\left(1+2.179 x^{1 / 2}+14.57 x\right), \\
x \Delta d_{v}(x) & =-0.02257 x^{0.3429}(1-x)^{3.864}\left(1+35.47 x^{1 / 2}+28.97 x\right), \\
x \Delta s(x) & =-0.02520 x^{0.3669}(1-x)^{7.649}\left(1+3.656 x^{1 / 2}+19.50 x\right), \\
x \Delta G(x) & =532.3 x^{3.544}(1-x)^{6.879}\left(1-3.147 x^{1 / 2}+3.148 x\right) .
\end{aligned}
$$

LSS'05 (Set 1) - NLO(JET) PD $\left(g_{1} / F_{1}\right)$ :

$$
\begin{aligned}
x \Delta u_{v}(x) & =0.4635 x^{0.6272}(1-x)^{3.428}\left(1+2.179 x^{1 / 2}+14.57 x\right), \\
x \Delta d_{v}(x) & =-0.02322 x^{0.3539}(1-x)^{3.864}\left(1+35.47 x^{1 / 2}+28.97 x\right), \\
x \Delta s(x) & =-0.02867 x^{0.4973}(1-x)^{7.649}\left(1+3.656 x^{1 / 2}+19.50 x\right), \\
x \Delta G(x) & =5.080 x^{0.9692}(1-x)^{6.879}\left(1-3.147 x^{1 / 2}+3.148 x\right) .
\end{aligned}
$$

LSS'05 (Set 2) - LO PD $\left(g_{1}+\mathrm{HT}\right)$ :

$$
\begin{aligned}
x \Delta u_{v}(x) & =0.1761 x^{0.3012}(1-x)^{3.177}(1+1.607 x)\left(1-0.4085 x^{1 / 2}+17.60 x\right), \\
x \Delta d_{v}(x) & =-0.00807 x^{0.1535}(1-x)^{3.398}(1+3.184 x)\left(1+37.25 x^{1 / 2}+31.14 x\right), \\
x \Delta s(x) & =-0.04464 x^{0.3239}(1-x)^{8.653}\left(1-0.9052 x^{1 / 2}+11.53 x\right), \\
x \Delta G(x) & =1.164 x^{0.4536}(1-x)^{5.511}\left(1-4.255 x^{1 / 2}+7.274 x\right) .
\end{aligned}
$$

LSS'05 (Set 2) - NLO $(\overline{\mathrm{MS}}) \operatorname{PD}\left(g_{1}+\mathrm{HT}\right)$ :

$$
\begin{aligned}
x \Delta u_{v}(x) & =0.4958 x^{0.6606}(1-x)^{3.428}\left(1+2.179 x^{1 / 2}+14.57 x\right), \\
x \Delta d_{v}(x) & =-0.02567 x^{0.3936}(1-x)^{3.864}\left(1+35.47 x^{1 / 2}+28.97 x\right), \\
x \Delta s(x) & =-0.02756 x^{0.3472}(1-x)^{7.649}\left(1+3.656 x^{1 / 2}+19.50 x\right), \\
x \Delta G(x) & =421.9 x^{2.949}(1-x)^{6.879}\left(1-3.147 x^{1 / 2}+3.148 x\right) .
\end{aligned}
$$


LSS'05 (Set 2) - NLO(JET) PD $\left(g_{1}+\mathrm{HT}\right)$ :

$$
\begin{aligned}
x \Delta u_{v}(x) & =0.4924 x^{0.6571}(1-x)^{3.428}\left(1+2.179 x^{1 / 2}+14.57 x\right), \\
x \Delta d_{v}(x) & =-0.02549 x^{0.3909}(1-x)^{3.864}\left(1+35.47 x^{1 / 2}+28.97 x\right), \\
x \Delta s(x) & =-0.02115 x^{0.3301}(1-x)^{7.649}\left(1+3.656 x^{1 / 2}+19.50 x\right), \\
x \Delta G(x) & =1009.5 x^{3.820}(1-x)^{6.879}\left(1-3.147 x^{1 / 2}+3.148 x\right) .
\end{aligned}
$$

\section{References}

[1] EMC, J. Ashman et al., Phys. Lett. B 206 (1988) 364; Nucl. Phys. B 328 (1989) 1.

[2] R. D. Carlitz, J. C. Collins and A.H. Mueller, Phys. Lett. B 214 (1988) 229; M. Anselmino, A. V. Efremov and E. Leader, Phys. Rep. 261 (1995) 1; H.-Y. Cheng, Int. J. Mod. Phys. A 11 (1996) 5109; D. Müller and O. V. Teryaev, Phys. Rev. D 56 (1997) 2607.

[3] SLAC E142 Coll., P.L. Anthony et al., Phys. Rev. D 54 (1996) 6620; SLAC/E154 Coll., K. Abe et al., Phys. Rev. Lett. 79 (1997) 26; SMC, B. Adeva et al., Phys. Rev. D 58 (1998) 112001; HERMES, K. Ackerstaff et al., Phys. Lett. B 404 (1997) 383; ibid B 442 (1998) 484; SLAC E143 Coll., K. Abe et al., Phys. Rev. D 58 (1998) 112003; SLAC/E155 Coll., P.L. Anthony et al., Phys. Lett. B 463 (1999) 339, ibid B 493 (2000) 19.

[4] JLab/Hall A Coll., X. Zheng et al., Phys. Rev. Lett. 92 (2004) 012004.

[5] E. Leader, A.V. Sidorov and D.B. Stamenov, Eur. Phys. J. C 23 (2002) 479.

[6] E. Leader, A.V. Sidorov and D.B. Stamenov, Phys. Rev. D 67 (2003) 074017.

[7] J. Blumlein and A. Tkabladze, Nucl. Phys. Proc. Suppl. 79 (1999) 541; S. Simula, M. Osipenko, G. Ricco and M. Taiuti, Phys. Rev. D 65 (2002) 034017.

[8] S. Wandzura and F. Wilczek, Phys. Lett. B 72 (1977) 195.

[9] E. Leader, A.V. Sidorov and D.B. Stamenov, in Particle Physics at the Start of the New Millennium, edited by A.I. Studenikin, World Scientific, Singapore, May 2001, p. 76. (Proceedings of the 9th Lomonosov Conference on Elementary Particle Physics, Moscow, Russia, 20-26 Sep 1999). 
[10] E. Leader, A. V. Sidorov and D. B. Stamenov, in Deep Inelastic Scattering DIS2003, edited by V.Kim and L.Lipatov, PNPI RAS, 2003, pp. 790-794, hep$\mathrm{ph} / 0309048$.

[11] O. Nachtmann, Nucl. Phys. B 63 (1973) 237.

[12] S. Wandzura, Nucl. Phys. B 122 (1977) 412; S. Matsuda and T. Uematsu, Nucl. Phys. B 168 (1980) 181.

[13] NMC Coll., M. Arneodo et al., Phys. Lett. B 364 (1995) 107.

[14] SLAC/E143 Coll., K. Abe et al., Phys. Lett. B 452 (1999) 194.

[15] A.D. Martin, R.G. Roberts, W.J. Stirling and R.S. Thorne, Eur. Phys. J. C 4 (1998) 463.

[16] A.D. Martin, R.G. Roberts, W.J. Stirling and R.S. Thorne, Eur. Phys. J. C 14 (2000) 133.

[17] Particle Data Group, Eur. Phys. J. C 15 (2000) 695.

[18] Asymmetry Analysis Collaboration, Y. Goto et al., Phys. Rev. D 62 (2000) 034017.

[19] G. Altarelli, S. Forte and G. Ridolfi, Nucl. Phys. B 534 (1998) 277; S. Forte, M. L. Mangano and G. Ridolfi, Nucl. Phys. B 602 (2001) 585.

[20] V. Barone, C. Pascaud and F. Zomer, Eur. Phys. J. C 12 (2000) 243.

[21] A.D. Martin, R.G. Roberts, W.J. Stirling and R.S. Thorne, Eur. Phys. J. C 28 (2003) 455.

[22] D0 Coll., B. Abbot et al., Pys. Rev. Lett. 86 (2001) 1707; CDF Coll., T. Affolder et al., Phys. Rev. D 64 (2001) 032001.

[23] A.W. Shreiber and A.W. Thomas, Phys. Lett. B 215 (1988) 141; R.D. Jaffe and A. Manohar, Nucl. Phys. B 337 (1990) 509.

[24] S.N. Shore and G. Veneziano, Phys. Lett. B 244 (1990) 75; S. Forte and E.V. Shuryak, Nucl. Phys. B 357 (1991) 153.

[25] A.E. Dorokhov, Czech. J. Phys. 52 (2002) c79; A.E. Dorokhov, N.I. Kochelev and Yu.A. Zubov, Int. Journ. Mod. Phys. A 8 (1993) 603; A.E. Dorokhov, N.I. Kochelev, Phys. Lett. B 304 (1993) 167. 
[26] M. Osipenko et al., Phys. Rev. D 71 (2005) 054007.

[27] I.I. Balitsky, V.M. Braun and A.V. Kolesnichenko, Phys. Lett. B 242 (1990) 245. Erratum ibid B318 (1993) 648; E. Stein et al., Phys. Lett. B 353 (1995) 107.

[28] J. Balla, M.V. Polyakov and C. Weiss, Nucl. Phys. B 510 (1998) 327; A.V. Sidorov and C. Weiss, hep-ph/0410253.

[29] E. Stein, Nucl. Phys. Proc. Suppl. 79 (1999) 567.

[30] COMPASS Coll., E.S. Ageev et al., hep-ex/0501073.

[31] M. Glück, E. Reya, M. Stratmann and W. Vogelsang, Phys. Rev. D 63 (2001) 094005.

[32] J. Blumlein, H. Bottcher, Nucl. Phys. B 636 (2002) 225.

[33] Asymmetry Analysis Collaboration, M. Hirai et al., Phys. Rev. D 69 (2004) 054021.

[34] M. Glück, E. Reya and A. Vogt, Eur. Phys. J. C 5 (1998) 461.

[35] S. Forte, hep-ph/0502073. 